Open Access to Pharmaceutical and Medical Research
(C) 2011-18, publisher and licensee JDDT, This is an Open Access article which permits
unrestricted non-commercial use, provided the original work is properly cited

Open@Access

Research Article

\title{
Pryazolylpyridine and Triazolylpyridine derivative of hydroxychloroquine as Potential Therapeutic against COVID-19: Theoretical Evaluation
}

\author{
Ruwida M.K. Omar*1, Adel M. Najar², Eman Bobtaina1, Awad F. Elsheikh ${ }^{3}$ \\ ${ }^{1}$ Department of Pharmaceutical chemistry, Benghazi University, Libya \\ 2 Department of Chemistry, Benghazi University, Libya \\ ${ }^{3}$ Depatment of Applied Research, research and manufacturing Agency, Benghazi, Libya
}

\begin{abstract}
In attempt to improve the biological activity of the well-known drug hydroxychloroquine (HQC), eight derivatives (HQ1-4Py - HQ8-4Py) based on the core of HQC were design and their electronic properties including frontier molecular orbitals, total energy and structural parameters were estimated at semi-empirical PM3 levels. Pharmacological parameters such as physicochemical, pharmacokinetics, drug-likeness and medicinal chemistry friendliness have been evaluated to estimate the drugs similarity. Introducing these moieties affect both electronic and drug likeness properties, HQ5-Py shows promised properties such large E $_{\mathrm{g}}$ and good clogP, The obtained results show that the suggested derivates may represent a potential drug candidate for COVID-19.
\end{abstract}

Keywords: Molecular similarity, COVID-19, Hydroxychloroquine (HCQ).

Article Info: Received 21 June 2020; Review Completed 18 July 2020; $\quad$ Accepted 29 July 2020; Available online 16 August 2020

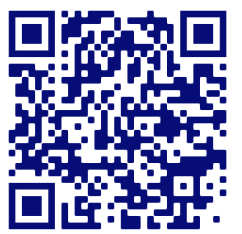

Cite this article as:

Omar RMK, Najar AM, Bobtaina E, Elsheikh AF, Pryazolylpyridine and Triazolylpyridine derivative of hydroxychloroquine as Potential Therapeutic against COVID-19: Theoretical Evaluation, Journal of Drug Delivery and Therapeutics. 2020; 10(4-s):181-186 http://dx.doi.org/10.22270/jddt.v10i4-s.4298

Ruwida M.K. Omar, Department of Pharmaceutical chemistry, Benghazi University, Libya

\section{INTRODUCTION}

In December 2019, a novel severe respiratory syndrome known as COVID-19caused by the infection with SARS-CoV-2 virus first broke out in Wuhan,China and subsequently spread tragically worldwide.1,2 On March 11, 2020 it was declared by WHO as a pandemic.3 Approximately $13,+27,440$ infections and 593,369 deaths worldwide up to July 18, 2020 have been recorded and the number of COVID-19 cases is still on the rise. However, the possible explanation of the rapid and serious deterioration is cytokine release syndrome (CRS) called Cytokine storm and overproduction of immune cells that cause the rapid failure of vital systems in the human body and fetal damages to tissues.

There is an urgent need to identify effective and safe $\operatorname{drug}(\mathrm{s})$ that can target the virus and/or modulate the immune response. ${ }^{4}$. Chloroquine (CQ) is a synthesized anti-malarial drug with immunomodulatory effects, has been recommended by many researchers after exhibiting the promising inhibitory effect of SARS-CoV-2. This was supported by clinical studies conducted on infected patients.5, 6 Hydroxychloroquine (HQC) an analogue of chloroquine as demonstrated in Figure 1. is considered as one of the disease-modifying anti-rheumatic drugs used to treat rheumatic diseases with $40 \%$ less toxicity than CQ in animals. HCQ had been tested in vitro against SARS-CoV-2 and demonstrated inhibitory effect for virus, 7 which suggest that it might be a potential pharmacological agent in treatment of COVID-19.8 Although the mechanism of action of both drugs is not clearly elucidated in COVID-19, but researches suggested that both CQ and HCQ are basic compounds, therefore, they elevate the $\mathrm{pH}$ of intracellular organelles essential for viral membrane fusion. 9,10 In addition, they could inhibit the SARS-CoV-2 permeation entry to the cell through changing the glycosylation of ACE2 receptor and spiking proteins. However, studies proposed that HCQ might be better than CQ in treatment of COVID-19 due to its ability to attenuate the acute progression of the infection. ${ }^{11}$ It also confers the same antiviral activity in both pre- and post-infection stages, finally it has fewer side effects ${ }^{12}$, safer profile in pregnancy, cheaper and more readily available.

Computer-Aided drug design (CADD) is an essential tool in drug discovery; these tools have a tremendous effect on both 
time and cost of drug developments. This includes the estimation of the electronic, drug-likeness, pharmacokinetic, 3D-QSAR, and physicochemical properties of the target candidates.

Herein, we report the electronic properties including electronic reactivity descriptors, molecular electrostatic potential surfaces (MEPS), and electrostatic potential, also geometrical parameters such as bond lengths, angles, and dihedral angle conformation were reported and discussed. Pharmacologically, parameters such as physicochemical properties, pharmacokinetics, drug-likeness and medicinal chemistry friendliness are also reported and analyzed for new designed molecules close to hydroxychloroquine.

Moreover, the rigidity and flexibility of molecules may affect the features of the target biological activity of designed molecules ${ }^{13}$. However, molecules with several rotatable bonds may adopt much different geometry, some of them being favourable due to the low internal energies while the others are not. Thus, in our designed molecules we have been considered couple of features in order to get molecules close to hydroxychloroquine biologically and electronically. Actually, the hybridized molecules which have more than biologically active moieties were targeted as a continuation of our research interest in the design, synthesis, investigation of molecular similarity of novel hybridized molecules; $\mathrm{N}$ heterocyclic based moieties ${ }^{14}$. Nowadays, drugs consist of quinolone such as QC and HCQ attracted more attention due to their activity against COVID-1915. Apparently, the main difference in chemical structure between chloroquine and hydroxychloroquine (Figure 1) as anti-malarial drugs is the presence of OH-group in hydroxychloroquine. However, from chemistry point view, the $\mathrm{OH}^{-}$increase the non-covalent interaction through it is ability to form hydrogen bonding that may enhance the biological activity hence the anti-viral activity.

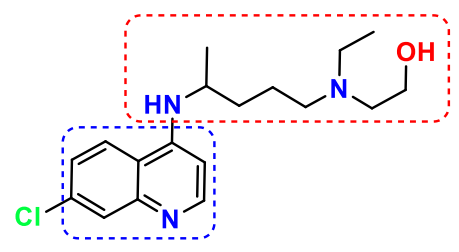

Hydroxychloroquine (HCQ)

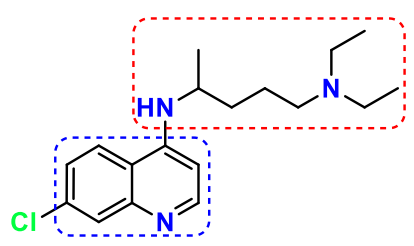

chloroquine (CQ)

Figure 1: Chemical structure of Hydroxychloroquine (HCQ) and Chloroquine (CQ)

\section{COMPUTATIONAL METHODS}

Semi-empirical PM3 quantum chemical calculations were conducted using HyperChem 8.0 Molecular Modeling program with root mean square (RMS) gradient $0.1 \mathrm{kcal} / \AA$ mol using Polak Ribiere algorithm ${ }^{16}$, all compounds were optimized at the mention level followed by frequency calculation to ensure that all compounds are minima with no imaginary frequency. SwissADME web tool has been used to compute the pharmacological parameters ${ }^{17}$.

\section{RESULTS AND DISCUSSION}

\section{Semi-empirical calculation}

In biological system, the non-covalent interaction forces are the essential forces for molecular functionality. So the designed molecules have ability to form: (i) $\pi$ - $\pi$-stacking; (ii) Hydrogen bonding; (iii) excellent coordinating site, and (iv) hydrophobic interaction 18 . All of the above non-covalent interaction are essential to hold supramolecular species together. ${ }^{18}$ However, self-assembly can be described as 'a process in which two or more components can spontaneously associate to form a larger, more-ordered structure through non-covalent interactions'. Generally, noncovalent interactions are very important in biological systems ${ }^{18,19}$ and the crystal structure of HQC reported its ability to form some of the non-covalent interaction such as hydrogen bonding and $\pi$ - $\pi$-stacking 20 .

Figure 3 shows the chemical structure of designed molecules. However, the compounds designed using 7chloroquinoline as core moiety and hydroxyl group. Moreover biological active units such as triazoles and others have been attached to the core unit to produce the candidate molecules. Molecules can be classified based on the connectivity to the 7-chloroqinioline into two classes (i) flexible molecules with methylene bridge between the core and the substituent and (ii) rigid molecules where the core is attached directly to the substituent. The flexible molecules have high degree of movement through methylene group which may increase the solubility. The rigid molecule which connected directly to core, could increase the interactions with the active site of the protein. Notably, semi-empirical PM3 calculation method was used based on the excellent agreement of some geometric parameters such as bond lengths and bond angles (Table 1) between the single crystal crystallography and calculated data of HCQ.

Molecular modeling program permits to build and analyze different molecular structures and determine the molecular, electronic, and biological properties. After setting Semiempirical MP3 method geometry optimization has been used to optimize the molecular structure to obtain a configuration characterized by a minimum free energy ${ }^{21}$. Within the geometry optimization the molecular symmetry calculated and preformed both molecular symmetry and asymmetry properties $^{22}$. However, all of calculation parameters such as total energy, dipole moment, binding energy, the HOMO, LUMO and others of candidate compounds reported based on optimal geometry. Moreover, the thermo-physical and electronic properties of designed molecules and hydroxychloroquine were listed in Table 2. Notably, the flexible molecules where unit 4-(1,2,3-triazol-4-yl)pyridin-2ol or 4-(pyrazol-3-yl)pyridin-2-ol connected to the main unit (7-chlrorquinioline) through - $\mathrm{CH} 2-$ reported thermophysical values fingerprinting to parent molecule HCQ. Additionally, same observations have been reported of electronic properties. It should be noted that, the flexibility of pyrazole/pyridine and triazole/pyridine offer a suitable space for non-covalent interaction, which enhance new drugs functionality. The compound coded HQ1-4Py used as an example; the total energy, binding energy and HOMO/LUMO energy gap for molecule calculated to be $83428.19 \mathrm{Kcal} / \mathrm{mol},-4089.28 \mathrm{Kcal} / \mathrm{mol}$ and $8.086 \mathrm{eV}$ respectively; whereas for $\mathrm{HQC}$ were $-83743.27 \mathrm{Kcal} / \mathrm{mol}$,$4888.10 \mathrm{Kcal} / \mathrm{mol}$ and $8.0928 \mathrm{eV}$. The chemical reactivity of 
molecules can be indicated from molecular orbitals. Although, HOMO/LUMO orbital indicate the electrophilic and nucleophilic attraction region in molecule as well as there is inverse relationship between $E_{\text {gap }}$ and molecular chemical reactivity 21 .

The chemical structure of HQC (Figure2) with atoms numbering. The Single Crystal X-Ray Crystallography data such as bond lengths and bond angles of HCQ were used to verified the selected method ${ }^{20}$. However, some geometric parameters of calculated and experimental HCQ molecule have been presented in Table 1 . The bond angle measured in (o) and bond length in $(\AA)$. Interestingly, excellent agreement between calculated and experimental data has been observed.<smiles>Oc1cc(-c2cn(Cc3ccnc4cc(Cl)ccc34)nn2)ccn1</smiles>

4-(1-((7-chloroquinolin-4-yl)methyl)-1 H-1,2,3-triazol4-yl)pyridin-2-ol<smiles>Oc1cc(-c2cnn(-c3ccnc4cc(Cl)ccc34)n2)ccn1</smiles><smiles>COCCN(CCCC(C)Nc1ccnc2cc(Cl)ccc12)C(=O)PC</smiles>

Figure 2: The structure of HCQ with atoms number

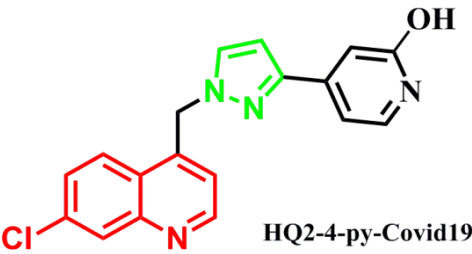

4-(1-((7-chloroquinolin-4-yl)methyl)-1 $H$-pyrazol-3-yl)<smiles>CC(C)C(O)C(C)C</smiles>

4-(1-(7-chloroquinolin-4-yl)-1H-pyrazol-3-yl) pyridin-2-ol

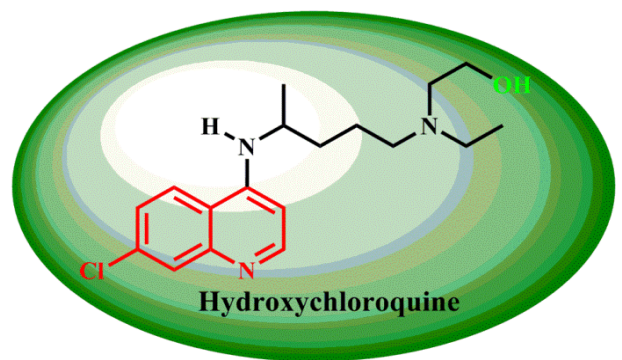<smiles>Oc1cnccc1-c1cn(Cc2ccnc3cc(Cl)ccc23)nn1</smiles>

4-(1-((7-chloroquinolin-4-yl)methyl)-1H-1,2,3triazol-4-yl)pyridin-3-ol<smiles>Oc1cnccc1-c1cn(-c2ccnc3cc(Cl)ccc23)nn1</smiles>

4-(1-(7-chloroquinolin-4-yl)-1 H-1,2,3triazol-4-yl)pyridin-3-ol

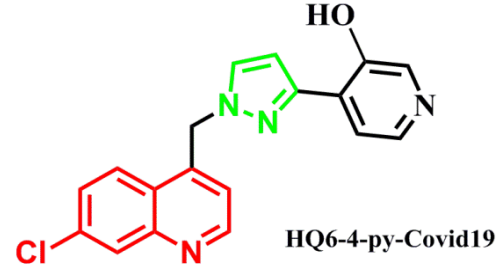

4-(1-((7-chloroquinolin-4-yl)methyl)-1 $H$-pyrazol-3-yl) pyridin-3-ol<smiles>Oc1cnccc1-c1ccn(-c2ccnc3cc(Cl)ccc23)n1</smiles>

4-(1-(7-chloroquinolin-4-yl)-1 $H$-pyrazol-3-yl) pyridin-3-ol

Figure 3: Chemical structure of hydroxychloroquine and new proposed derivatives 
Table 1: Selected experimental and calculated bond lengths and angles

\begin{tabular}{llllll} 
& $\begin{array}{l}\text { Exp. Bond } \\
\text { length }\end{array}$ & $\begin{array}{l}\text { Calc. Bond } \\
\text { length }\end{array}$ & $\begin{array}{l}\text { Exp. Bond } \\
\text { angles }\end{array}$ & $\begin{array}{l}\text { Calc. Bond } \\
\text { angles }\end{array}$ \\
\hline $\mathrm{N}(1)-\mathrm{C}(7)$ & 1.30 & 1.32 & $\mathrm{~N}(1)-\mathrm{C}(7)-\mathrm{C}(6)$ & 124.6 & 122.32 \\
$\mathrm{C}(6)-\mathrm{C}(5)$ & 1.45 & 1.38 & $\mathrm{C}(\mathrm{l})-\mathrm{N}(1)-\mathrm{C}(7)$ & 120.7 & 119.35 \\
$\mathrm{C}(16)-\mathrm{O}(1)$ & 1.41 & 1.41 & $\mathrm{C}(5)-\mathrm{C}(2)-\mathrm{C}(10)$ & 124.6 & 117.46 \\
$\mathrm{~N}(2)-\mathrm{C}(10)$ & 1.48 & 1.49 & $\mathrm{C}(15)-\mathrm{C}(16)-0$ & 113.6 & 106.8 \\
$\mathrm{Cl}-\mathrm{C}(2)$ & 1.74 & 1.70 & $\mathrm{~N}(3)-\mathrm{C}(17)-\mathrm{C}(18)$ & 113.3 & 111.18
\end{tabular}

Bond angle ( ${ }^{\circ}$ ) and Bond length $(\AA)$

Table 2: Some calculated electronic and thermos-physical properties of HCQ and designed molecules

\begin{tabular}{ccccccccc} 
& \multicolumn{3}{c}{ Thermo-physical properties } & \multicolumn{2}{l}{ Electronic properties } \\
& E & B. energy & $\begin{array}{l}\text { Dipole } \\
\text { (Debye) }\end{array}$ & mom. & Elumo $(\mathrm{eV})$ & $\begin{array}{l}\text { Eномо } \\
(\mathrm{eV})\end{array}$ & $\begin{array}{l}\text { Energy } \\
(\mathrm{eV})\end{array}$ & gap \\
HQ1-4Py & -83428.19 & -4089.28 & 3.505 & -1.324 & -9.41 & 8.086 \\
HQ2-4Py & -82785.54 & -4214.82 & 5.453 & -1.201 & -9.27 & 8.069 \\
HQ3-4Py & -79973.90 & -3803.03 & 4.136 & -1.668 & -9.339 & 7.671 \\
HQ4-4Py & -79336.43 & -3933.74 & 3.760 & -1.489 & -9.118 & 7.629 \\
HQ5-4Py & -83422.30 & -4083.40 & 4.296 & -1.266 & -9.281 & 8.015 \\
HQ6-4Py & -82781.55 & -4210.84 & 3.840 & -1.117 & -9.15 & 8.04 \\
HQ7-4Py & -79970.70 & -3799.82 & 3.18 & 1.513 & -9.142 & 7.629 \\
HQ8-4Py & -79330.96 & -3928.27 & 2.16 & -1.317 & -8.891 & 7.574 \\
HCQ & -83743.27 & -4888.10 & 3.653 & -0.7872 & -8.88 & 8.0928
\end{tabular}

Total energy (T. energy), Binding energy (B. energy), dipole moment (dipole mom.)

\section{Biological activity evaluation}

In term of calculated QSAR parameters as indication of biological activity the cLog $\mathrm{P}$ value of hydroxychloroquine agreed with some of new designed molecules. Table 3 shows some QSAR calculation values of proposed compounds and hydroxychloroquine. All findings confirm that the molecules are hydrophobic to some extent. However, the closeness of calculated Log P value between HQC and HQ1-4Py has been observed at value 4.20 for both molecules suggesting that
HQ1-4Py and HCQ might have same biological activity; as the cLog P values plays as essential role for determination of molecular biological reactivity 21 as well as the hydrophobicity often used to predict pharmacokinetics and pharmacodynamics ${ }^{23}$. The closed molecules in reactivity to hydroxychloroquine were HQ1-4Py, HQ2-4Py, HQ5-4Py, HQ6-4Py. The reactivity values of HQC was 97.93 whereas for HQ1-4Py; 92.10, HQ2-4Py; 92.24, HQ5-4Py; 91.76 and HQ6-4Py; 91.89.

Table 3: Calculated QSAR parameters of designed molecules and hydroxychloroquine

$\begin{array}{llllllll} & \text { P. charge } & \text { S. } \operatorname{area}(\mathrm{Ap}) & \text { S. } \text { area(Gr) } & \text { Volume } & \text { Polarizability } & \text { Reactivity } & \begin{array}{l}\text { cLog } \\ \text { P }\end{array} \\ \text { HQ1-4Py } & 0 & 411.20 & 547.37 & 903.63 & 36.59 & 92.10 & 4.20 \\ \text { HQ2-4Py } & 0 & 426.38 & 555.88 & 918.02 & 37.30 & 92.24 & 4.65 \\ \text { HQ3-4Py } & 0 & 387.01 & 509.68 & 847.79 & 34.75 & 87.27 & 4.10 \\ \text { HQ4-4Py } & 0 & 398.241 & 512.121 & 855.963 & 35.46 & 87.40 & 4.55 \\ \text { HQ5-4Py } & 0 & 397.84 & 543.45 & 901.28 & 36.59 & 91.76 & 3.96 \\ \text { HQ6-4Py } & 0 & 413.13 & 551.92 & 918.02 & 37.30 & 91.89 & 4.41 \\ \text { HQ7-4Py } & 0 & 371.41 & 507.31 & 843.40 & 34.75 & 86.92 & 3.87 \\ \text { HQ8-4Py } & 0 & 378.41 & 502.92 & 849.49 & 35.46 & 87.06 & 4.32 \\ \text { HQC } & 0 & 580.13 & 618.84 & 1030.66 & 38.78 & 97.93 & 3.49\end{array}$

Partial charge; (P.charge), Surface area (Approx); S.area (Ap), Surface Area(Grid); S.area (Gr) 


\section{Physicochemical and Pharmacokinetic Analysis}

A comprehensive analysis of the drug-likeness properties of new hybridized molecules was performed and results were compared to HCQ by using SwissADME web tool. However, the proposed structures were qualified as candidates, the compounds molecular properties of Lipinski's rule of five. were calculated. The number of hydrogen bond acceptors and donors for all compounds were in accordance with Lipinski`s rule of five. The molecular weights of all compounds were within the range. The lipophilicity property (MLogP $\leq 4.15$, octanol-water partition coefficient) was in the range for all compounds and the results are shown in Table 4.

Table 4: Some Important Computed Physicochemical Properties of the newly eight designed molecules compared to HCQ

\begin{tabular}{llllllll} 
Compound & Mwt & nRot & HBA & TPSA & M LogP & Ali Log S & MR \\
HQ1-4-py & 337.76 & 3 & 5 & 76.72 & 2.09 & -4.02 & 91.34 \\
HQ2-4-py & 336.78 & 3 & 4 & 63.83 & 2.32 & -4.31 & 93.54 \\
HQ3-4-py & 323.74 & 2 & 5 & 76.72 & 2.12 & -4.08 & 86.92 \\
HQ4-4-py & 322.75 & 2 & 4 & 63.83 & 2.36 & -4.38 & 89.13 \\
HQ5-4-py & 337.76 & 3 & 5 & 76.72 & 1.68 & -3.67 & 91.34 \\
HQ6-4-py & 336.78 & 3 & 4 & 63.83 & 1.92 & -3.96 & 93.54 \\
HQ7-4-py & 323.74 & 2 & 5 & 76.72 & 1.71 & -3.74 & 86.92 \\
HQ8-4-py & 322.75 & 2 & 4 & 63.83 & 1.95 & -4.03 & 89.13 \\
\hline
\end{tabular}

Optimal range: molecular weight (MW) $\leq 600$, lipophilicity log or Moriguchi octane-water partition coefficient (M log P) $\leq 5$, aqueous solubility descriptor (Ali $\log \mathrm{S}) \leq 0$, hydrogen-bonded acceptor $(\mathrm{HBA}) \leq 10$, topological polar surface area (TPSA) $\leq 150$ $\AA^{2}$, number of rotatable bonds $\left(\mathrm{n}_{\mathrm{Rot}}\right) \leq 10$, and molar refractivity $(\mathrm{MR}) \leq 155$

The medicinal chemistry was evaluated, and the results are shown in Table 5 confirmed the likeness of the eight hybridized molecules to hydroxychloroquine with acceptable synthetic accessibility.

Table 5: Medicinal chemistry evaluation of the hybrid compounds

\begin{tabular}{lcccc} 
Compounds & PAINS Alert & Brenk alert & Lead likeness & $\begin{array}{c}\text { Synthetic } \\
\text { accessibility }\end{array}$ \\
HQ1-4-py & 0 & 0 & yes & 2.65 \\
HQ2-4-py & 0 & 0 & yes & 2.5 \\
HQ3-4-py & 0 & 0 & yes & 2.72 \\
HQ4-4-py & 0 & 0 & yes & 2.49 \\
HQ5-4-py & 0 & 0 & yes & 2.68 \\
HQ6-4-py & 0 & 0 & yes & 2.43 \\
HQ7-4-py & 0 & 0 & yes & 2.73 \\
HQ8-4-py & 0 & 0 & yes & 2.43 \\
\hline
\end{tabular}

Finally, Swiss ADME evaluated the pharmacokinetic properties of hybrids like, gastro-intestinal absorption, blood brain barrier permeability, inhibition the cytochrome P450 isoenzymes (CYP isoenzymes), and substrate Pglycoprotein $\mathrm{g} \mathrm{24}$. As demonstrated below in Table 6 all hybrids presented high gastro-intestinal absorption and being permeable to blood brain barrier. They also were (yes) to all CYP except HQ3-4-py and HQ7-4-py which did not inhibit CYP2C9. Eventually, they all could substrate Pgp except compounds coded HQ1-4-py, HQ3-4-py, HQ4-4-py and HQ7-4-py.

Table 6: Pharmacokinetic evaluation of hybrids (GI: gastro-intestinal absorption, BBB: blood brain barrier, CYP: Cytochromes, P-gp: P-glycoprotein

$\begin{array}{lcccc}\text { Compounds } & \text { GI absorption } & \text { BBB perment } & \text { Pgp Substrate } & \text { CYPX Inhibition } \\ \text { HQ1-4-py } & \text { High } & \text { Yes } & \text { No } & \text { Yes } \\ \text { HQ2-4-py } & \text { High } & \text { Yes } & \text { Yes } & \text { Yes } \\ \text { HQ3-4-py } & \text { high } & \text { Yes } & \text { No } & \text { Yes* } \\ \text { HQ4-4-py } & \text { High } & \text { Yes } & \text { yos } & \text { Yes } \\ \text { HQ5-4-py } & \text { High } & \text { yes } & \text { Yes } & \text { Yes } \\ \text { HQ6-4-py } & \text { High } & \text { Yes } & \text { No } & \text { Yes } \\ \text { HQ7-4-py } & \text { High } & \text { Yes } & \text { Yes } & \text { Yes } \\ \text { HQ8-4-py } & \text { High } & \text { Yes } & \end{array}$




\section{CONCLUSION}

In the present work in silico screening procedures including Semi-empirical MP3 calculation method and SwissADME web tool were used for comparison eight hybrid chemical structures with the parent compound hydroxychloroquine. Results have demonstrated excellent agreement between single crystal X-ray cystography data of HCQ and calculated data. However, we conclude that the flexible molecules recorded values closed to HCQ drug. Computationally, molecule coded HQ1-4Py reported identical electronic and thermo-physical values to HCQ. The electronic parameters such as HOMO/LUMO energy gap reflect that HQ1-4Py an HCQ may have same stability as well as may have same molecular reactivity. Moreover, some QSAR parameters of designed molecules reordered values in good agreement with HCQ, which may have same biological activity. The HQ54 Py and HCQ recorded Log P values at 3 which reveal that the two molecules may have same molecular biological also may have same pharmacokinetics and pharmacodynamics properties. However, based on the geometric comparison of HQC; it has been noted that the calculated Log P values using HyperChem software may more accurate than what has been recorded using SwissADME tool.

\section{REFERENCES}

[1] J. Gao, Z. Tian, and X. Yang, "Breakthrough: Chloroquine phosphate has shown apparent efficacy in treatment of COVID-19 associated pneumonia in clinical studies," Biosci. Trends, 2020.

[2] Organization WH. Coronavirus disease (COVID-2019) situation reports. Geneva: WHO; 2020.

[3] Organization WH. Coronavirus disease (COVID-19) advice for the public. 2020 .

[4] NIH U.S. National Library of Medicine. Available at: https://clinicaltrials.gov/. Accessed April 42020.

[5] A. H. J. Kim et al., "A Rush to Judgment? Rapid Reporting and Dissemination of Results and Its Consequences Regarding the Use of Hydroxychloroquine for COVID-19," Ann. Intern. Med., 2020.

[6] I. Ben-Zvi, S. Kivity, P. Langevitz, and Y. Shoenfeld, "Hydroxychloroquine: From malaria to autoimmunity," Clinical Reviews in Allergy and Immunology. 2012.

[7] X. Yao et al., "In Vitro Antiviral Activity and Projection of Optimized Dosing Design of Hydroxychloroquine for the Treatment of Severe Acute Respiratory Syndrome Coronavirus 2 (SARS-CoV-2)," Clin. Infect. Dis., 2020.

[8] K. A. Pastick et al., "Review : Hydroxychloroquine and Chloroquine for Treatment of SARS-CoV-2 ( COVID-19)," vol.
2, pp. 1-9, 2019

[9] "Sanofi-Aventis U.S. LLC. Aralen Chloroquine Phosphate, USP, 2013.

[10] Concordia Pharmaceuticals Inc. Plaquenil Hydroxychloroquine Sulfate Tablets, USP, 2017.

[11] C. Biot et al., "Design and synthesis of hydroxyferroquine derivatives with antimalarial and antiviral activities," J. Med. Chem., 2006.

[12] U.S. Food and Drug Administration. EUA Hydroxychloroquine sulfate Health Care Provider Fact Sheet March 28, 2020.

[13] J.P. T. Bultinck, Patrick, Winter, Hans DeWi If ried Langenaeker, Ed., Computational Medical Chemistry for Drug discovery. NEW YORK: Taylor \& Francis Group LLC, 2004.

[14] K. V. Luna Parada LK, Vargas Méndez LY, “QuinolineSubstituted 1,2,3-Triazole-Based Molecules, As Promising Conjugated Hybrids in Biomedical Research.," Org. Med. Chem IJ, vol. 7, no. 2, pp. 001-0010.

[15] J. Liu et al., "Hydroxychloroquine, a less toxic derivative of chloroquine, is effective in inhibiting SARS-CoV-2 infection in vitro," Cell Discovery. 2020.

[16] W. F. Coleman and C. R. Arumainayagam, "HyperChem 5 (by Hypercube, Inc.)," J. Chem. Educ., 1998.

[17] A. Daina, O. Michielin, and V. Zoete, "SwissADME: A free web tool to evaluate pharmacokinetics, drug-likeness and medicinal chemistry friendliness of small molecules," Sci. Rep., 2017.

[18] J. W. Steed, D. R. Turner, and K. J. Wallace, Core Concepts in Supramolecular Chemistry and Nanochemistry. 2007.

[19] D. L. Caulder and K. N. Raymond, "Supermolecules by Design," Acc. Chem. Res., vol. 32, no. 11, pp. 975-982, Nov. 1999.

[20] A. Semeniuk, J. Kalinowska-Tluscik, W. Nitek, and B. J. Oleksyn, "Intermolecular Interactions in Crystalline Hydroxychloroquine Sulfate in Comparison with Those in Selected Antimalarial Drugs," J. Chem. Crystallogr., vol. 38, no. 5, pp. 333-338, 2008.

[21] M. J. Islam, A. Zannat, A. Kumer, N. Sarker, and S. Paul, "The prediction and theoretical study for chemical reactivity, thermophysical and biological activity of morpholinium nitrate and nitrite ionic liquid crystals: A DFT study," Adv. J. Chem. A, vol. 2, no. 4, pp. 316-326, 2019.

[22] M. J. Islam, N. Sarker, and A. Kumer, "The evaluation and comparison of thermo-physical, chemical and biological properties of palladium (II) complexes on binuclear diamine ligands with different anions using the DFT method," Int. J. Adv. Biol. Biomed. Res., vol. 7, no. 4, pp. 315-334, 2019.

[23] S. Ekins, Computer Applications in Pharmaceutical Research and Development. 2006.

[24] C. A. Lipinski, F. Lombardo, B. W. Dominy, and P. J. Feeney, "Experimental and computational approaches to estimate solubility and permeability in drug discovery and development settings," Advanced Drug Delivery Reviews. 1997. 